\title{
Hydrogen at zinc vacancy of ZnO: an EPR and ESEEM study
}

Nguyen Tien Son, J. Isoya, Ivan Gueorguiev Ivanov, T. Ohshima and Erik Janzén

Linköping University Post Print

Tweet

N.B.: When citing this work, cite the original article.

Original Publication:

Nguyen Tien Son, J. Isoya, Ivan Gueorguiev Ivanov, T. Ohshima and Erik Janzén, Hydrogen at zinc vacancy of ZnO: an EPR and ESEEM study, 2014, INTERNATIONAL CONFERENCE ON DEFECTS IN SEMICONDUCTORS 2013, 341-344.

http://dx.doi.org/10.1063/1.4865666

Copyright: (C) 2014 AIP Publishing LLC

http://scitation.aip.org/

Postprint available at: Linköping University Electronic Press

http://urn.kb.se/resolve?urn=urn:nbn:se:liu:diva-111622 


\section{AIP | Proceedings}

\section{Hydrogen at zinc vacancy of ZnO: An EPR and ESEEM study}

N. T. Son, J. Isoya, I. G. Ivanov, T. Ohshima, and E. Janzén

Citation: AIP Conference Proceedings 1583, 341 (2014); doi: 10.1063/1.4865666

View online: http://dx.doi.org/10.1063/1.4865666

View Table of Contents: http://scitation.aip.org/content/aip/proceeding/aipcp/1583?ver=pdfcov

Published by the AIP Publishing

\section{Articles you may be interested in}

Size effects on formation energies and electronic structures of oxygen and zinc vacancies in $\mathrm{ZnO}$ nanowires: A first-principles study

J. Appl. Phys. 109, 044306 (2011); 10.1063/1.3549131

Zinc and oxygen vacancies in $\mathrm{ZnO}$ nanorods

J. Appl. Phys. 106, 104307 (2009); 10.1063/1.3259413

Further characterization of oxygen vacancies and zinc vacancies in electron-irradiated $\mathrm{ZnO}$

J. Appl. Phys. 103, 043710 (2008); 10.1063/1.2833432

Identification of oxygen and zinc vacancy optical signals in $\mathrm{ZnO}$

Appl. Phys. Lett. 89, 262112 (2006); 10.1063/1.2424641

Oxygen vacancies in $\mathrm{ZnO}$

Appl. Phys. Lett. 87, 122102 (2005); 10.1063/1.2053360 


\title{
Hydrogen at zinc vacancy of ZnO: an EPR and ESEEM study
}

\author{
N. T. Son ${ }^{\mathrm{a}}$, J. Isoya ${ }^{\mathrm{b}}$, I. G. Ivanov ${ }^{\mathrm{a}}$, T. Ohshima ${ }^{\mathrm{c}}$, and E. Janzén ${ }^{\mathrm{a}}$ \\ ${ }^{a}$ Department Physics, Chemistry and Biology, Linköping University, SE-58183 Linköping, Sweden \\ ${ }^{b}$ Graduate School of Library, Information \& Media Studies, University of Tsukuba, Tsukuba, Ibaraki 305-8550, \\ Japan \\ ¿Japan Atomic Energy Agency, Takasaki, Gunma 370-1292, Japan
}

\begin{abstract}
An electron paramagnetic resonance (EPR) spectrum, labeled S1, with small-splitting doublet accompanied by weak satellites is observed in $\mathrm{ZnO}$ irradiated with $2 \mathrm{MeV}$ electrons. The obtained structure is shown to be the hyperfine structure due to the dipolar interaction between an unpaired electron spin and a nuclear spin of hydrogen $(\mathrm{H})$. The observation of the nuclear Zeeman frequency of $\mathrm{H}$ in electron spin echo envelope modulation experiments further confirmed the presence of a hydrogen atom in S1. From the observed spin-Hamiltonian parameters, S1 is identified to be the partly $\mathrm{H}$-passivated $\mathrm{Zn}$ vacancy, $\mathrm{V}_{\mathrm{Zn}}^{-} \mathrm{H}^{+}$, with the $\mathrm{H}^{+}$ion making a short $\mathrm{O}-\mathrm{H}$ bond with only one nearest $\mathrm{O}$ neighbor of $\mathrm{V}_{\mathrm{Zn}}$ in the basal plane, being off the substitutional site, while the unpaired electron spin, which gives rise to the observed EPR signal, is localized on the $p$ orbital of another O neighbor also in the basal plane.
\end{abstract}

Keywords: $\mathrm{ZnO}$, hydrogen, vacancies, hyperfine interaction, electron paramagnetic resonance.

PACS: 71.55.Gs, 76.30.Lh, 61.72.Hh, 61.72.jd

\section{INTRODUCTION}

Hydrogen is known to be present in $\mathrm{ZnO}$ grown by any method. Calculations predicted that the formation energy of the interstitial $\mathrm{H}^{+}$is low enough to allow a large concentration of $\mathrm{H}$ in as-grown $\mathrm{ZnO} .{ }^{1}$ In the bond-center configurations, $\mathrm{H}_{\mathrm{BC}}$, hydrogen interstitials act as shallow donors and have been suggested to be the source for the natural $\mathrm{n}$-type doping in as-grown $\mathrm{ZnO} .{ }^{1}$ The $\mathrm{H}_{\mathrm{BC}}$ centers were later identified by infrared (IR) spectroscopy. ${ }^{2,3}$ Hydrogen-related shallow donors have also been identified by muon spin rotation ${ }^{4}$ and electron paramagnetic resonance (EPR). ${ }^{5}$ The isolated $\mathrm{H}$ at an $\mathrm{O}$ vacancy, $\mathrm{H}_{\mathrm{O}}$, was predicted to bond equally with all nearest $\mathrm{Zn}$ neighbors, forming a multi-bond center ${ }^{6}$ or to occur simply as an anion $\mathrm{H}^{-}$at the anion $\mathrm{O}$ site. ${ }^{7}$ The prediction on $\mathrm{H}_{\mathrm{O}}$ was later supported by IR absorption. ${ }^{3}$ The isolated $\mathrm{H}$ interstitials are predicted to have low migration energies $(\sim 0.5 \mathrm{eV}$ for different configurations of $\mathrm{H}_{\mathrm{BC}}$ ) and can become mobile at room temperature. ${ }^{8}$ More thermally stable H-related defects are therefore expected to be $\mathrm{H}$ interstitials trapping at acceptor impurities ${ }^{9,10,11}$ or interstitial $\mathrm{H}_{2}$ molecules. ${ }^{12}$ The $\mathrm{H}$ passivation of intrinsic acceptors, such as the $\mathrm{Zn}$ vacancy $\left(\mathrm{V}_{\mathrm{Zn}}\right)$ in $n$-type $\mathrm{ZnO}$, is also predicted to be highly energetically favorable, forming the partly $\left(\mathrm{V}_{\mathrm{Zn}} \mathrm{H}\right)$ and fully $\left(\mathrm{V}_{\mathrm{Zn}} \mathrm{H}_{2}\right) \mathrm{H}$-passivated $\mathrm{V}_{\mathrm{Zn}}$ defects. ${ }^{8}$ The $\mathrm{V}_{\mathrm{Zn}} \mathrm{H}_{2}$ complex was suggested to be the defect model for the H-II center observed by IR spectroscopy. ${ }^{2}$ In EPR studies of electronirradiated $\mathrm{ZnO}$ by Evans and co-workers ${ }^{13}$ the doublets $\mathrm{D}$ and $\mathrm{E}$ were suggested to be related to the axial and nonaxial configurations of $\mathrm{V}_{\mathrm{Zn}}^{-} \mathrm{H}^{+}$.

In the present study, a new EPR doublet spectrum is observed in electron-irradiated $\mathrm{ZnO}$. From the spinHamiltonian parameters observed by EPR, the doublet is identified to be the $\mathrm{V}_{\mathrm{Zn}}^{-} \mathrm{H}^{+}$defect. The involvement of a $\mathrm{H}$ atom in the defect is further confirmed by the observation of the nuclear Zeeman frequency of ${ }^{1} \mathrm{H}$ by electron spin echo envelope modulation (ESEEM). The microscopic model of the defect is discussed.

\section{EXPERIMENT}

Samples used in this study are nominally undoped $\mathrm{ZnO}$ substrates from Tokyo Denpa which have the resistivity in the range of $\sim 500-1000 \Omega \mathrm{cm}$. The irradiation by $2 \mathrm{MeV}$ electrons was performed at room temperature to doses $2-4 \times 10^{18} \mathrm{~cm}^{-2}$. Continuous-wave EPR measurements were performed on a Bruker ELEXSYS E500 X-band ( 9.45 $\mathrm{GHz}$ ) spectrometer. Electron spin echo (ESE) detected EPR and ESEEM experiments were performed on a Bruker ELEXSYS E580 X-band $(\sim 9.75 \mathrm{GHz})$ spectrometer. Both spectrometers are equipped with Oxford continuous He-

International Conference on Defects in Semiconductors 2013

AIP Conf. Proc. 1583, 341-344 (2014); doi: 10.1063/1.4865666

(C) 2014 AIP Publishing LLC 978-0-7354-1215-6/\$30.00 
flow cryostats, allowing the regulation of the sample temperature in the range of 4-295 K. In the pulsed experiments, the echo intensity was obtained by integrating the echo signal within the range of the gate centered on the top of the echo. By changing the width of the gate, either non-selective detection, which collects responses from all the signals within the excitation bandwidth of the microwave pulses, or a selective detection, which virtually eliminates the contribution from off-resonance signals, is chosen. In the echo-detected EPR, the intensity of Hahn-echo $\left(90^{\circ}-\right.$ $\tau-180^{\circ}-\tau-$ echo) was measured as a function of the external magnetic field strength B. For ESEEM measurements, a similar two-pulse sequence was used and the echo intensity was measured as a function of time.

\section{RESULTS AND DISCUSSION}

Figure 1 shows the EPR spectra in $\mathrm{ZnO}$ after irradiation by $2 \mathrm{MeV}$ electrons at room temperature to a dose of $2 \times 10^{18} \mathrm{~cm}^{-2}$ measured in darkness at $20 \mathrm{~K}$. For the magnetic field $\mathbf{B}$ along the $c$ axis $(\mathbf{B} \| \mathbf{c})$, the spectrum shows a dominating doublet, labelled $\mathrm{S} 1$, and weak signals of the $\mathrm{S}$ center ${ }^{14}$ and the negative $\mathrm{Zn}$ vacancy, $\mathrm{V}_{\mathrm{Zn}}^{-}$, in non-axial configuration $^{15}$ and $\mathrm{Mn}^{2+}$ (Ref. 16) [Fig. 1(a)]. The doublet splits into six doublets when rotating the magnetic field away from the $c$ axis as can be seen in the spectrum measured at an angle $\theta \sim 65^{\circ}$ [ Fig. 1(b)]. Four doublets, labeled $1,3,4$ and 6 , have weak satellites with a similar splitting of $\sim 10.5 \mathrm{G}$. These satellites are not observed for doublets 2 and 5 [Fig. 1(b)]. The appearance of weak satellites and the variation of the intensity between the doublets are typical features of a system having an electron spin $S=1 / 2$ and a nuclear spin $I=1 / 2$ with the nuclear Zeeman interaction comparable to the hyperfine (hf) interaction. This has been observed for shallow impurities having large nuclear g-values, such as the phosphorous shallow donors in $4 \mathrm{H}-$ and $6 \mathrm{H}-\mathrm{SiC} .{ }^{17}$ The satellites are related to the forbidden transitions of the system $\mathrm{S}=1 / 2, \mathrm{I}=1 / 2$, which become partly allowed when the relative strength between the hf interaction and the nuclear Zeeman interaction changes. The splitting of the forbidden lines corresponds to the two times of the nuclear Zeeman frequency $\left(2 v_{\mathrm{N}}\right)$ of the involved impurity. In this case, $2 v_{\mathrm{N}} \sim 10.5 \mathrm{G}$ or $\sim 29.43 \mathrm{MHz}$ which is very close to the two times nuclear Zeeman frequency of ${ }^{1} \mathrm{H}\left[2 v_{\mathrm{N}}(\mathrm{H}) \sim 29.80 \mathrm{MHz}\right.$ at $3500 \mathrm{G}$ or $\sim 28.52$ $\mathrm{MHz}$ at $3350 \mathrm{G}]$. This indicates that the $\mathrm{S} 1$ doublet is related to $\mathrm{H}$.

We found that the angular dependence of the doublet S1, including the forbidden lines, with the magnetic field rotating in the $(11 \overline{2} 0)$ plane can be well described by the spin-Hamiltonian

$$
\mathrm{H}=\mu_{\mathrm{B}} \text { B.g.S }+ \text { S.A.I }-\mathrm{g}_{\mathrm{n}} \mu_{\mathrm{n}} \mathbf{B} . \mathbf{I} .
$$

Here the first term describes the electronic Zeeman interaction, the second term represents the $\mathrm{hf}$ interaction and the

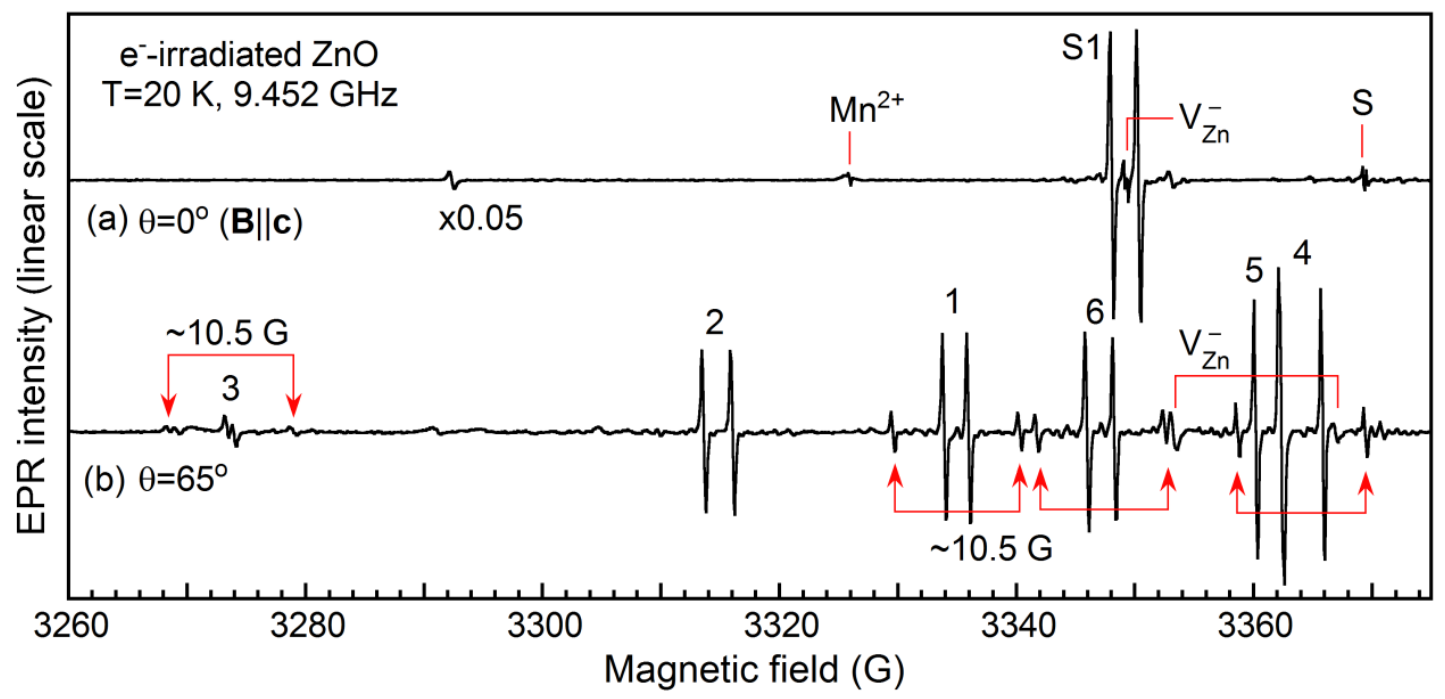

FIGURE 1. (Color online) EPR spectra of S1 in electron-irradiated $\mathrm{ZnO}$ measured in darkness at $20 \mathrm{~K}$ for (a) $\mathbf{B} \| \mathbf{c}$ and (b) $\mathbf{B}$ is in the $(11 \overline{2} 0)$ plane and $\sim 65^{\circ}$ away from the $c$ axis, showing the doublet structure with the splitting varying in the range of $\sim 2.4-3.5$ G. At an arbitrary angle of the magnetic field, the doublet is split into six ones. All the doublets, except the ones labeled 2 and 5 , have two weak satellites with a splitting in the range of $\sim 10.5 \mathrm{G}$. The field modulation is $0.2 \mathrm{G}$. The intensity of the spectrum in

(a) is reduced by 20 times 
Table 1. The g- and A-tensor, their principal values and the polar angle $\theta$ and azimuthal angle $\varphi$ of the principal axes, of the S1 center in $\mathrm{ZnO}$. The angles $\theta=0$ and $\varphi=0$ correspond to the [0001] and [11 $\overline{2} 0]$ directions, respectively. The principal A-values are given in $10^{-4} \mathrm{~cm}^{-1}$ (the corresponding values in MHz using the conversion factor $\mathrm{A}[\mathrm{MHz}]=2.99792453684 \mathrm{~A}\left[10^{-4} \mathrm{~cm}^{-1}\right]$ are also given in parentheses) while $\theta$ and $\varphi$ and are measured in degrees. The maximum errors in determination of the principal gand A-values are \pm 0.0003 and $\pm 0.2 \times 10^{-4} \mathrm{~cm}^{-1}$, respectively.

\begin{tabular}{|c|c|c|c|c|c|c|c|}
\hline \multirow{2}{*}{$\frac{\text { Parameters }}{\sigma}$} & \multicolumn{3}{|c|}{ Matrix elements } & \multicolumn{2}{|c|}{ Principal value } & \multirow{2}{*}{$\frac{\theta}{67.57}$} & \multirow{2}{*}{$\frac{\varphi}{353.43}$} \\
\hline & 2.0120 & 0.0146 & -0.0064 & 1 & 2.0077 & & \\
\hline & & 2.0464 & -0.0244 & 2 & 2.0648 & 117.42 & 71.07 \\
\hline & & & 2.0163 & 3 & 2.0023 & 36.73 & 117.02 \\
\hline \multirow[t]{3}{*}{$\mathbf{A}$} & -3.316 & -2.250 & -1.015 & 1 & $-4.4(13.2)$ & 78.30 & 21.15 \\
\hline & & 1.838 & -0.756 & 2 & $2.8(8.4)$ & 107.64 & 107.38 \\
\hline & & & 1.478 & 3 & $1.6(4.8)$ & 21.38 & 143.07 \\
\hline
\end{tabular}

last one is the nuclear Zeeman term. $\mathbf{g}$ and $\mathbf{A}$ are the g- and A-tensor, $\mu_{\mathrm{B}}$ and $\mu_{\mathrm{n}}$ are the Bohr and nuclear magneton, respectively, and $\mathrm{g}_{\mathrm{n}}$ is the nuclear $\mathrm{g}$ value for ${ }^{1} \mathrm{H}\left(\mathrm{g}_{\mathrm{n}}=5.5856912\right)$. It is noticed that the forbidden lines can only be fitted using the $\mathrm{g}_{\mathrm{n}}$ value for ${ }^{1} \mathrm{H}$. Thus, the involvement of a $\mathrm{H}$ atom in the $\mathrm{S} 1$ defect is unambiguously confirmed. The obtained spin-Hamiltonian parameters for S1 are given in Table 1.

In a system with an electron spin $\mathbf{S}$ and a nuclear spin $\mathbf{I}$, as in the case of $\mathbf{S 1}, \mathbf{S}$ induces an inductive field at the site of $\mathbf{I}$. A sudden change of the polarization of $\mathbf{S}$ (by the microwave field in ESEEM experiments) induces a change of the sign of this local field. The precession of $\mathbf{I}$ about the resultant nuclear precession field will then induce a change in the field at the site of $\mathbf{S}$. This acts as a field modulation, causing a slight difference in the precession frequency of the electron spin about the external magnetic field $\mathbf{B}$. Such a field modulation is known to have an effect on the ESE spectrum which can be detected by ESEEM. ${ }^{18}$ From ESEEM experiments, the nuclear Zeeman frequency can be directly deduced in a similar way as in electron nuclear double resonance (ENDOR) experiments. ${ }^{19}$

We performed ESEEM experiments on the weak doublet 3 in Fig. 1(b). Fig. 2 shows the frequency-domain ESEEM spectrum obtained from Fourier transform of the time-domain ESEEM spectrum measured at $20 \mathrm{~K}$. As can be seen in the figure, four frequencies, labeled 1-4, are observed. Here, lines 2 and 3 correspond to the allowed transitions with the frequencies $\left|v_{\mathrm{N}}-\mathrm{A} / 2\right|$ and $\left|v_{\mathrm{N}}+\mathrm{A} / 2\right|$ with $\mathrm{A}$ is the hf splitting and $v_{\mathrm{N}}$ the nuclear Zeeman frequency, while lines 1 and 4 correspond to the difference (A) and the sum $\left(2 v_{\mathrm{N}}\right)$ of the two allowed frequencies, respectively. Thus, line 4 corresponds to $2 v_{\mathrm{N}} \sim 30.91 \mathrm{MHz}$. This value is close to the two times of the nuclear Zeeman frequency of ${ }^{1} \mathrm{H}\left(2 v_{\mathrm{N}}(\mathrm{H}) \sim 29.80 \mathrm{MHz}\right.$ at $\left.3500 \mathrm{G}\right)$. This observation thus unambiguously confirms the involvement of a hydrogen atom in the S1 defect, further supporting the EPR identification. Also, the involvement of any impurity with $\mathrm{I} \neq 0$ and a reasonable natural abundance of a few percentages or larger, such as Li or common transition metals, in S1 can be excluded (otherwise their nuclear Zeeman frequencies should be detected in ESEEM).

From the observed g-tensor, it is clear that the $\mathrm{S} 1$ defect has low symmetry $C_{1}$. This suggests that $\mathrm{H}$ is not at the

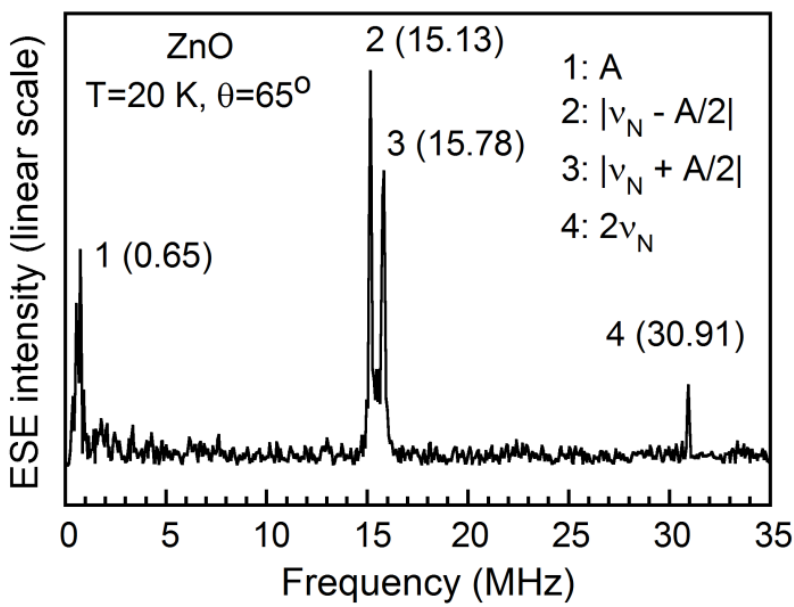

FIGURE 2. Two-pulse ESEEM frequency-domain spectrum measured at $20 \mathrm{~K}$ on doublet 3 of S1 in Fig. 1(b) at the angle $\theta \sim$ $65^{\circ}$ from the $c$ axis $(\mathrm{B}=3365.76 \mathrm{G}$ at the microwave frequency of $9.72029 \mathrm{GHz})$ showing the allowed and forbidden transitions. The frequencies are shown in parentheses in MHz. 
substitutional site. The very small hf constants, as shown in Table 1, and the traceless hf A-tensor (i.e., $\mathrm{A}_{1}+\mathrm{A}_{2}+\mathrm{A}_{3}$ $=0$ ) indicate that $\mathrm{H}$ does not carry the electron spin and the hf interaction is a pure dipolar interaction with the axis of the dipole along the axis of $A_{1}\left[A_{\|}=A_{1}\right.$, and $\left.A_{\perp}=\left(A_{2}+A_{3}\right) / 2\right]$. This means that the unpaired electron should be localized on the $p$ orbital of an atom having a nuclear spin $\mathrm{I}=0$. It is likely that the unpaired electron is localized at an $\mathrm{O}$ atom, since the only $\mathrm{O}$ isotope having $\mathrm{I} \neq 0$ is ${ }^{17} \mathrm{O}(\mathrm{I}=5 / 2)$ which has too low natural abundance $(0.038 \%)$ to be detectable. (If the unpaired electron spin is on a $\mathrm{Zn}$ atom, then the hf interaction with ${ }^{67} \mathrm{Zn}$ ( $\mathrm{I}=5 / 2$ and $4.1 \%$ natural abundance) should give rise to a detectable hf structure.) Moreover, the S1 center is observed after electron irradiation which creates high concentrations of vacancies. Therefore, the best defect model for $\mathrm{S} 1$ is a positive $\mathrm{H}^{+}$ ion trapped at a negative $\mathrm{Zn}$ vacancy $\mathrm{V}_{\mathrm{Zn}}^{-}$. The observed Euler angles of the dipole (along the axis of $\mathrm{A}_{1}$ ) $\theta \sim 78^{\circ}$

with the $c$ axis and $\varphi \sim 21^{\circ}$ with the $<11 \overline{2} 0>$ direction suggest that the unpaired electron should be on one of three $\mathrm{O}$ atoms in the basal plane, while $\mathrm{H}$ makes a short $\mathrm{O}-\mathrm{H}$ bond with another $\mathrm{O}$ neighbor $\mathrm{V}_{\mathrm{Zn}}$ also in the basal plane, forming a low symmetry $C_{1}$ defect.

The $\mathrm{D}$ and $\mathrm{E}$ centers was previously assigned to the axial and non-axial configurations of $\mathrm{V}_{\mathrm{Zn}}^{-} \mathrm{H}^{+}$, respectively, by Evans and co-workers. ${ }^{13}$ The assignment of $\mathrm{D}$ as a H-related center was based only on the observation of two weak satellites with a splitting of $\sim 11 \mathrm{G}$, which is close to the two times of the nuclear Zeeman frequency of ${ }^{1} \mathrm{H}$. However, neither the symmetry nor parameters of these two centers were determined (only a single EPR spectrum measured at the $c$ direction was reported) ${ }^{13}$ The E center was observed in all our samples, while the $\mathrm{D}$ doublet could not be detected in the whole temperature range of 5-295 K (neither in darkness nor under illumination). It is noticed that in darkness the $\mathrm{E}$ spectrum can only be detected at temperatures above $50 \mathrm{~K}$ when the $\mathrm{S} 1$ signal disappears. Due to lack of data, it is not clear whether the $\mathrm{E}$ center is also related to $\mathrm{H}$ and if it can be a candidate for the axial configuration or an excited state of the $\mathrm{V}_{\mathrm{Zn}}^{-} \mathrm{H}^{+}$defect.

In summary, we have observed an EPR doublet, S1, in electron-irradiated ZnO. It is suggested from EPR and ESEEM experiments that $\mathrm{S} 1$ is a positive $\mathrm{H}^{+}$ion trapped at an $\mathrm{O}$ neighbor of a negative $\mathrm{Zn}$ vacancy $\mathrm{V}_{\mathrm{Zn}}^{-}$in the basal plane, making a short $\mathrm{O}-\mathrm{H}$ bond, while the unpaired electron spin is localized on the $p$ orbital of another $\mathrm{O}$ neighbor of $\mathrm{V}_{\mathrm{Zn}}$ also in the basal plane, forming a low symmetry $C 1$ defect.

\section{ACKNOWLEDGMENTS}

Support from the Linköping Linnaeus Initiative for Novel Functional Materials (VR) and the Knut and Alice Wallenberg Foundation is gratefully acknowledged.

\section{REFERENCES}

1. C. G. Van de Walle, Phys. Rev. Lett. 85, 1012-1015 (2000).

2. E. V. Lavrov, J. Weber, F. Börrnert, C. G. Van de Walle, and R. Helbig, Phys. Rev. B 66, 165205 (2002).

3. E. V. Lavrov, F. Herklotz, and J. Weber, Phys. Rev. B 79, 165210 (2009).

4. S. F. J. Cox, E. A. Davis, S. P. Cottrell, P. J. C. King, J. S. Lord, J. M. Gil, H. V. Alberto, R. C. Vilão, J. Piroto Duarte, N. Ayres de Campos, A. Weidinger, R. L. Lichti, and S. J. C. Irvine, Phys. Rev. Lett. 86, 2601-2604 (2001).

5. D. M. Hofmann, A. Hofstaetter, F. Leiter, H. Zhou, F. Henecker, B. K. Meyer, S. Orlinskii, J. Schmidt, and P. G. Baranov, Phys. Rev. Lett. 88, 045504 (2002).

6. A. Janotti and C. G. Van de Walle, Nat. Mater. 6, 44-47 (2007).

7. H. Takenaka and D. J. Singh, Phys. Rev. B 75, 241102(R) (2007).

8. M. G. Wardle, J. P. Goss, and P. R. Briddon, Phys. Rev. Lett. 96, 204504 (2006).

9. E. V. Lavrov, F. Herklotz, and J. Weber, Phys. Rev. B 79, 165210 (2009).

10. G. A. Shi, M. Stavola, and W. B. Fowler, Phys. Rev. B 73, 081201(R) (2006).

11. S. J. Jokela and M.D. McCluskey, Phys. Rev. B 76, 193201 (2007).

12. G. A. Shi, M. Saboktakin, M. Stavola, and S.J. Pearton, Appl. Phys. Lett. 85, 5601-5603 (2004).

13. S. M. Evans, N. C. Giles, L. E. Halliburton, and L. A. Kappers, J. Appl. Phys. 103, 043710 (2008).

14. D. Galland and A. Herve, Phys. Lett. 33A, 1-2 (1970).

15. B. Schallenberger and A. Hausmann, Z. Phys. B 23, 177-181 (1976).

16. P. B. Dorain, Phys. Rev. 112, 1058-1060 (1958).

17. N. T. Son, A. Henry, J. Isoya, M. Katagiri, A. Gali, and E. Janzén, Phys. Rev. B 73, 075201 (2006).

18. L. G. Rowan, E. L. Hahn, and W. B. Mims, Phys. Rev. 137, A61-A71 (1965).

19. J. Isoya, M. K. Bowman, J. R. Norris, and J. A. Weil, J. Chem. Phys. 78, 1735-1746 (1983). 